\title{
Diffusion-Weighted Imaging: An Exciting and Problem-Solving Tool in Patients with Hepatic Metastases
}

\author{
Savia Gupta ${ }^{1}$ Naseer A. Choh ${ }^{1}$ Mohd Gull Bhatt ${ }^{2}$ \\ ${ }^{1}$ Department of Radiodiagnosis, Sher-i-Kashmir Institute of Medical \\ Sciences, Srinagar, Jammu and Kashmir, India \\ ${ }^{2}$ Department of Medical Oncology, Sher-i-Kashmir Institute of \\ Medical Sciences, Srinagar, Jammu and Kashmir, India \\ ${ }^{3}$ Department of General Surgery, Sher-i-Kashmir Institute of \\ Medical Sciences, Srinagar, Jammu and Kashmir, India \\ ${ }^{4}$ Department of Pathology, Sher-i-Kashmir Institute of Medical \\ Sciences, Srinagar, Jammu and Kashmir, India
}

\author{
Rauf A. Wani ${ }^{3}$ Zubaida Rasool $^{4} \quad$ Sheikh R. Rasool ${ }^{1}$
}

Address for correspondence Naseer Ahmad Choh, MBBS, MD, Department of Radiodiagnosis, Sher-i-Kashmir Institute of Medical Sciences, Srinagar, Jammu and Kashmir 7006311145, India (e-mail: savia6020@gmail.com).

\author{
Abstract \\ Keywords \\ - diffusion-weighted \\ imaging \\ - contrast-enhanced \\ computed \\ tomography \\ - hepatic metastases \\ - multiphase MRI \\ - ultrasonography
}

Background The diffusion-weighted imaging (DWI) is based on the random Brownian motion of water molecules that influences image contrast depending on different pathological conditions.

Objective The purpose of this study was to evaluate the efficacy of various magnetic resonance imaging (MRI) sequences including diffusion-weighted and gadobenate-enhanced MRI in the detection and characterization of liver lesions in a patient of known primary malignancy and to compare MRI with contrast-enhanced computed tomography (CECT) and ultrasonography (USG) in the detection of liver metastases.

Methods All patients underwent a multiphase MRI. The final diagnosis was established by histopathological examination.

Results A total of 43 patients of known primary malignancy were enrolled. MRI gave a provisional diagnosis of liver metastases in 21 patients and benign disease in 22 patients with histopathological correlation revealing two false-negative and one false-positive result. In the detection of lesions, DWI outscored other sequences ( 92.9 vs. $83.5 \%$ in hepatobiliary phase vs. $55.0 \%$ in $\mathrm{T}_{2}$-weighted sequences) with a statistically significant difference noted only in comparison with $\mathrm{T}_{2}$-weighted sequences $(p<0.001)$. In 16 patients, MRI added new lesions that were not detected by CECT/USG. The sensitivity and specificity of MRI for detecting metastases were 90.9\%/95.2\% and 97.9\%/96.8\% for per-patient and per-lesion basis, respectively.

Conclusion Multiphase MRI improved both the detection and characterization of liver metastases. Adding DWI to the routine MR sequences helped in detecting small liver metastases $(<10 \mathrm{~mm})$ not detected by other sequences. published online May 23, 2021
DOI https://doi.org/

$10.1055 / \mathrm{s}-0041-1729490$ ISSN 0971-3026
(C) 2021. Indian Radiological Association

This is an open access article published by Thieme under the terms of the Creative Commons Attribution-NonDerivative-NonCommercial-License, permitting copying and reproduction so long as the original work is given appropriate credit. Contents may not be used for commercial purposes, or adapted, remixed, transformed or built upon. (https://creativecommons.org/licenses/by-nc-nd/4.0/).

Thieme Medical and Scientific Publishers Private Ltd. A-12, Second Floor, Sector -2, NOIDA -201301, India 


\section{Introduction}

The liver is the most common organ to be involved with metastatic disease, common primary sites being colon, breast, lung, pancreas, and stomach. ${ }^{1}$ The detection, characterization, enumeration, and localization of metastatic hepatic neoplasms are critical for planning appropriate therapy. Though there are numerous noninvasive methods for the detection of the liver metastases, magnetic resonance imaging (MRI) is well recognized as one of the most sensitive methods for the detection of hepatic metastasis. ${ }^{2}$ The advent of diffusion-weighted imaging (DWI) and the hepatobiliary specific contrast agents (gadobenate-dimeglumine) has further increased the sensitivity of MRI., ${ }^{34}$ In this study, we evaluated the efficacy of contrast-enhanced MRI in detecting and characterizing liver lesions in patients with known primary malignancy.

\section{Materials and Methods}

The study was approved by the institutional ethical committee.

\section{Study Population}

A total of 43 patients of known primary cancer with liver lesions that were not characterized by contrast-enhanced computed tomography (CECT) and ultrasonography (USG) were included. Multiparametric MRI with contrast using gadobenate was done in all patients after taking proper written consent from patients or their first-degree relatives.

\section{MRI Technique}

MRI was performed on a 1.5T MR system (Magnetom Avanto, Siemens Medical System) Germany.

\section{Precontrast Imaging}

Axial breath-hold $\mathrm{T}_{1}$-weighted fast low angle shot (FLASH) 2D, $\mathrm{T}_{2}$-weighted turbo spin-echo (TSE) with and without fat suppression, axial breath-hold $\mathrm{T}_{1}$-weighted FLASH 2D in and out of phase, coronal and axial $\mathrm{T}_{2}$-weighted half-Fourier acquisition single-shot turbo spin-echo (HASTE) imaging of the liver were performed. Respiratory-triggered DW-MRI was performed using three $b$-values $\left(b=0\right.$ second $\mathrm{mm}^{2}, \mathrm{~b}=$ 400 second $\mathrm{mm}^{2}$, and $\mathrm{b}=800$ second $\mathrm{mm}^{2}$ ). Apparent diffusion coefficient $(A D C)$ values were measured by placing the region of interest on the lesion on the ADC map.

\section{Postcontrast Imaging}

Following intravenous gadobenate dimeglumine ( $0.1 \mathrm{mmol} / \mathrm{kg}$ using a power-injector followed by $20 \mathrm{~mL}$ saline flush), dynamic contrast-enhanced axial $\mathrm{T}_{1}$-weighted MRI with fat suppression was performed in arterial (13 seconds), portovenous (60 seconds), equilibrium (180 seconds), and hepatobiliary phase (60 minutes) of contrast enhancement using volume-interpolated breath-hold examination.

\section{Image Analysis}

Lesions were characterized as benign or metastatic based on a combination of signal intensity. Lesions with all the following characteristics, (a) hyperintense signal on $\mathrm{T}_{2}$-weighted imaging and long echo time $\mathrm{T}_{2}$-weighted imaging, (b) low signal on high $b$ values with isointense signal compared with surrounding parenchyma on the ADC map, and (c) enhancement pattern characteristic of the hemangioma, were considered benign. A lesion with any or combination of the following features, (a) mild or moderate signal hyperintensity on $\mathrm{T}_{2}$-weighted images, (b) mild or moderate hypointensity on $\mathrm{T}_{1}$-weighted images with possible peripheral rim enhancement on dynamic phase and hypointense appearance on hepatobiliary phase whereas on DWI images, (c) hyperintense signal at low and high $\mathrm{b}$ values with corresponding low signal on ADC map with ADC values lower than that of surrounding liver parenchyma, were considered malignant. The characteristics of the lesions were confirmed by histopathology (biopsy/fine-needle aspiration cytology) wherever available. For subjects with multiple lesions, one lesion was analyzed histopathologically with similar appearing lesions accepted as either metastases or benign as per the histopathology report. Few lesions with definite/clear cut diagnosis on MRI (focal fat infiltration, cysts, perfusion defect) were not analyzed histopathologically.

The number of lesions detected on a particular sequence were counted individually. Then the total number of lesions detected by MRI were calculated that were taken as the maximum number of lesions detected on any of the sequences. A comparison was made between various MRI sequences and between CECT and USG for the metastatic lesion detection rate.

\section{Statistical Analysis}

Statistical analysis was performed using software package SPSS-22. Data were tabulated in mean \pm standard deviation, $\%$ age. The differences of the data were tested for statistical significance, that is, $p$-value was calculated. A $p$-value $<0.05$ was considered significant. Finally, the sensitivity and specificity of the MRI in detecting liver metastases were calculated on a per-patient and per-lesion basis.

\section{Results}

A total of 43 patients with known primary malignancy and liver lesions uncharacterized on other modalities were included in this prospective study. Demographically mean age of the study population was 51.2 years with males and females accounting for 21 and 22 patients, respectively.

\section{Lesion Characterization}

Totally, 21 patients had a tentative diagnosis of metastatic disease on MRI with histopathology affirming the diagnosis in 20. One patient with a tentative diagnosis of metastases was characterized as hemangioma on histopathology. In 22 patients with MRI tentative diagnosis of benign disease, 
10 patients had a definite diagnosis (focal fat infiltration $=$ 2 , cysts $=7$, perfusion defect $=1$ ) and did not require histopathological correlation (-Figs. 1 and 2). In the remaining 12 patients, hemangiomas were the most common lesion on histopathology (7) followed by alveolar hydatid (2) and sclerosed hemangioma (1). Two patients with MR diagnosis of hemangiomas were metastases on histopathology. The sensitivity and specificity of MRI on the per-patient basis in detecting metastases were 90.9 and 95.2\%, respectively (-Table 1).

\section{Lesion Detection}

In 22 patients with metastatic disease, the total number of lesions detected by MRI were 96 as compared with 37 and 47 detected on USG and CECT (- Table 2) with statistically significant differences noted between MRI and USG ( $p<$ $0.001)$ and between MRI and CECT $(p<0.001)$. When various MRI sequences were compared for the detection of the metastatic lesions, the maximum number of metastatic lesions were detected by DWI ( - Figs. 3 and $\mathbf{4}$ ) followed by hepatobiliary phase, and then portovenous phase and least number were detected by $\mathrm{T}_{2}$-weighted HASTE sequence ( $\mathbf{- T a b l e ~} \mathbf{3}$ ). In one patient, no lesion was seen on HASTE and precontrast $\mathrm{T}_{1}$-weighted weighted images, but significant number of lesions were seen on DWI and postcontrast $\mathrm{T}_{1}$-weighted images (-Figs. 5 and $\mathbf{6}$ ).
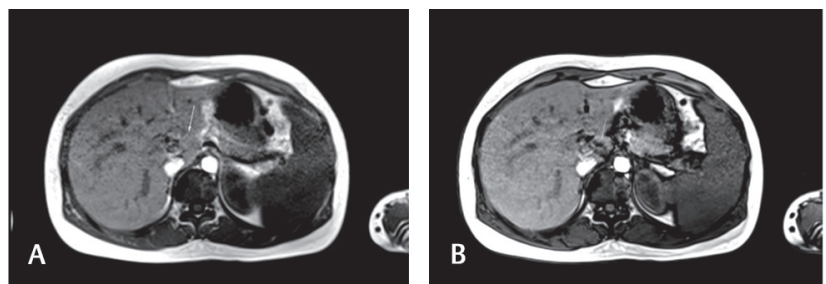

Fig. 1 A 35-year-old female with colon cancer: (A) T1-weighted in-phase image showing an isointense lesion in the caudate lobe and corresponding out-phase image (B) showing signal drop out(arrow) suggesting focal fat infiltration.

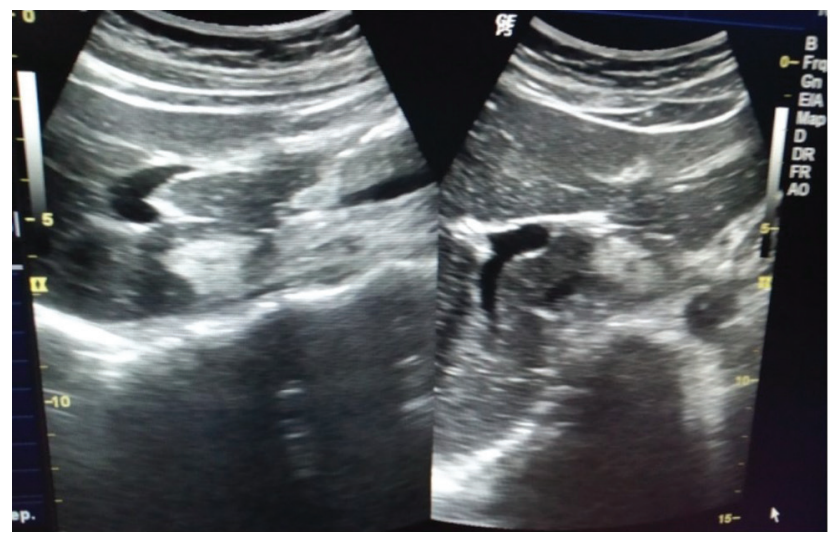

Fig. 2 Ultrasonography image of the same patient showing a hyperechoic lesion in the caudate lobe.
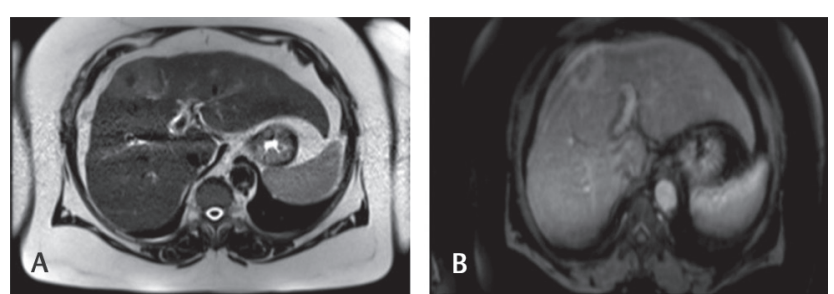

Fig. 3 A 55-year-old male with colon cancer: (A) T2-weighted half-Fourier acquisition single-shot turbo spin-echo and (B) venous phase postcontrast $\mathrm{T} 1$-weighted images showing a single lesion at segment VIII.
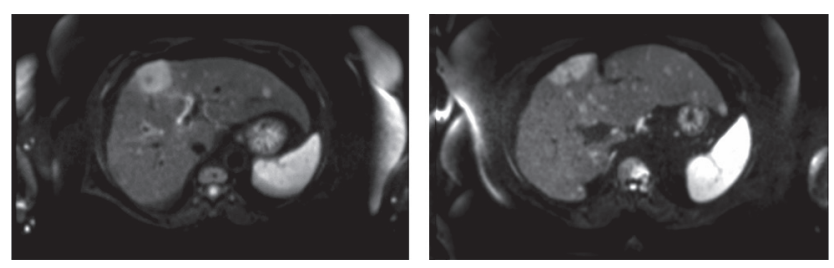

Fig. 4 Diffusion-weighted images with $b$ values of $b=400$ second $\mathrm{mm}^{2}$ in the same patient showing multiple small metastases not detected by other sequences.

Table 1 Comparison on the per-patient basis between MRI tentative and final diagnosis

\begin{tabular}{|l|l|l|l|}
\hline \multirow{2}{*}{ MRI tentative diagnosis } & Final diagnosis & \multirow{2}{*}{ Total } \\
\cline { 2 - 4 } & Benign & Metastases & \\
\hline Benign & 20 & 2 & 22 \\
\hline Metastases & 1 & 20 & 21 \\
\hline Total & 21 & 22 & 43 \\
\hline
\end{tabular}

Abbreviation: MRI, magnetic resonance imaging.

Table 2 Comparison between MRI, CECT, and USG in metastatic lesion detection rate

\begin{tabular}{|l|l|l|l|l|}
\hline \multicolumn{2}{|l|}{ Total number of metastatic lesions mean \pm SD } & Minimum & Maximum \\
\hline USG & 37 & $1.68+1.08$ & 0.0 & 4.0 \\
\hline CECT & 47 & $2.13+1.16$ & 1.0 & 5.0 \\
\hline MRI & 96 & $4.36 \pm 3.59$ & 1.0 & 11.0 \\
\hline
\end{tabular}

Abbreviations: CECT, contrast-enhanced computed tomography; MRI, magnetic resonance imaging; SD, standard deviation; USG, ultrasonography. 
Table 3 Comparison between various MRI sequences in terms of metastases detection rate

\begin{tabular}{|l|l|r|c|c|}
\hline MRI sequences & \multicolumn{1}{|c|}{$\begin{array}{c}\text { No. of metastatic lesions detected by a particular } \\
\text { sequence/Total metastatic lesions detected by MRI }\end{array}$} & \multicolumn{2}{|c|}{ Mean \pm SD } & \multicolumn{2}{|c|}{ Minimum } & \multicolumn{2}{|l|}{ Maximum } \\
\hline$T_{1}$-weighted & $55 / 96$ & $2.55 \pm 1.62$ & 6 \\
\hline $\begin{array}{l}T_{2} \text {-breath hold fat-suppressed } \\
\text { TSE }\end{array}$ & $44 / 96$ & $2.00 \pm 1.30$ & 1 \\
\hline$T_{2}$-HASTE & $42 / 96$ & $1.90 \pm 1.37$ & 0 \\
\hline DWI & $90 / 96$ & $4.00+3.27$ & 1 & 10 \\
\hline Portovenous phase & $70 / 96$ & $3.40+2.64$ & 1 \\
\hline Hepatobiliary phase & $76 / 96$ & $3.59+2.75$ & 1 \\
\hline
\end{tabular}

Abbreviations: DWI, diffusion-weighted imaging; T2-HASTE, T2-weighted half-Fourier acquisition single-shot turbo spin-echo; MRI, magnetic resonance imaging; SD, standard deviation; TSE, turbo spin-echo.
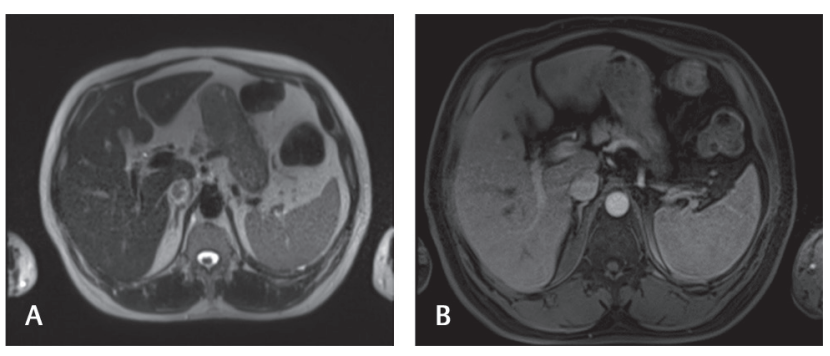

Fig. 5 A 65-year-old male with rectum cancer: (A) T2-weighted half-Fourier acquisition single-shot turbo spin-echo and (B) precontrast T1-weighted images showing no definite liver lesion.

\section{Discussion}

In our study, sensitivity, specificity, positive predictive, and negative predictive value of MRI on a per-patient basis for detecting metastatic lesions were 90.9, 95.2, 95.2, and 90.9\%, respectively. There were two false-negative results in which histopathologically proved metastases were mischaracterized as hemangiomas on MRI. The discordance could be because of the fact that low cellularity lesions may not show restricted diffusion. Both these lesions showed free diffusion with high ADC values $\left(1.410^{-3}\right.$ and $\left.1.42 \times 10^{-3} \mathrm{~mm}^{2} / \mathrm{s}\right)$ that overlapped with the mean ADC value of the hemangiomas $\left(1.37 \pm 0.2610^{-3} \mathrm{~mm}^{2} / \mathrm{s}\right)$. In one false-positive result with MRI findings of metastases, histopathology confirmed it to be a hemangioma. This might be because of the atypical imaging characteristics of hemangioma that often imitates metastases. Also, the ADC value of the lesion in this patient was $0.8410^{3} \mathrm{~mm}^{2} / \mathrm{sec}$ that overlapped with the mean ADC value of the metastatic lesions of our study $(0.89 \pm 0.17)$.

MRI detected 96 metastatic lesions, whereas CECT and USG detected 47 and 37 metastatic lesions, respectively, with a significant difference between them. Study results were concordant with the retrospective study of Kim et $\mathrm{al}^{5}$ in which MRI with DWI detected new lesions not detected by CT. Similarly, Muhi et al ${ }^{6}$ compared the diagnostic accuracy of CECT and contrast-enhanced MRI using hepatocyte-specific contrast agent in detecting hepatic metastases and found contrast-enhanced MRI to be more accurate than CECT. Similar results were also seen in the studies of Vreugdenburg et $\mathrm{al}^{7}$ and Hagspiel et $\mathrm{al}^{8}$ in which MRI was shown to be better than CECT at identifying and characterizing liver lesions. The majority of the additional lesions detected in our study were subcentimetric; this was consistent with the study of Ichikawa et $\mathrm{al}^{9}$ in which MRI was found to have higher sensitivity in detecting small lesions than CT.

Our study showed DWI to be superior to $\mathrm{T}_{2}$-weighted sequences ( $\mathrm{T}_{2}$-turbo spin-echo [TSE]) in detecting liver metastases. For lesions $>10 \mathrm{~mm}$ size, DWI detected 39 metastatic lesions, whereas $\mathrm{T}_{2}$-TSE detected 31 lesions. For lesions $<10 \mathrm{~mm}$ size, 51 metastatic lesions were detected by DWI, while $\mathrm{T}_{2}$-TSE detected 13 lesions only. When the analysis was performed according to the lesion size, a statistically significant difference was noted in the detection rate of DWI and $\mathrm{T}_{2}$-TSE for both types of metastatic lesions (lesions with size $<10 \mathrm{~mm}$ and lesions with size $>10 \mathrm{~mm}$ ), and the difference was highly significant $(p<0.0001)$ for lesions $<10 \mathrm{~mm}$ in which
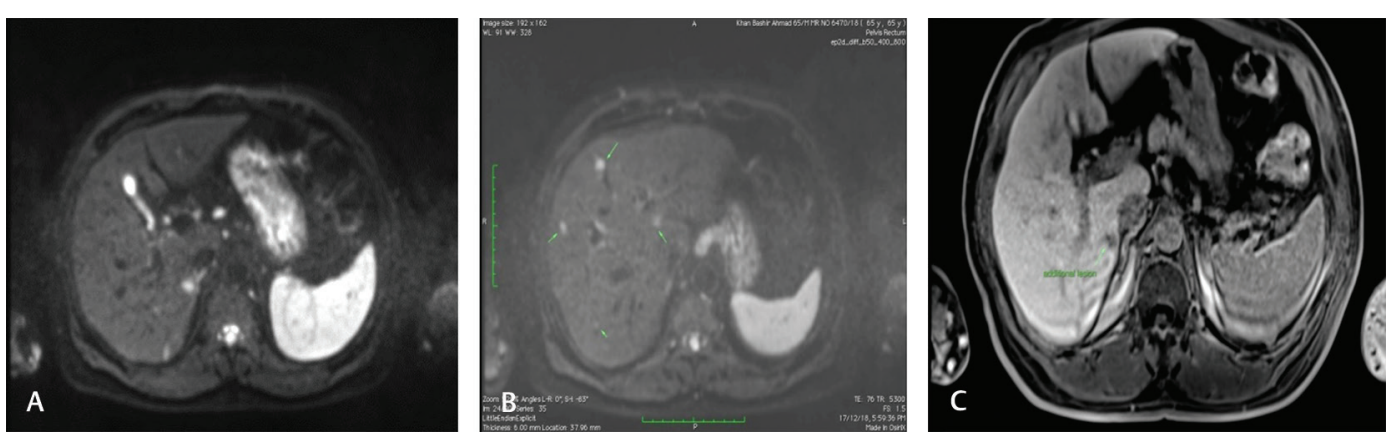

Fig. 6 (A and B) Diffusion-weighted (C) hepatobiliary phase images in the same patient showing lesions (arrow) not detected by T2-weighted half-Fourier acquisition single-shot turbo spin-echo and precontrast T1-weighted images (metastases). 
DWI detected 38 more lesions compared with $\mathrm{T}_{2}$-TSE. Our results were in line with the study of Bruegel et $\mathrm{al}^{10}$ in which they compared respiratory triggered diffusion-weighted imaging-echo-planar imaging (DWI-EPI) with $\mathrm{T}_{2}$-TSE. They found a sensitivity and specificity of 45 to $62 \%$ for $\mathrm{T}_{2}$-TSE MRI and 88 to $91 \%$ for DWI-EPI for small lesions. DWI was also found superior to precontrast $\mathrm{T}_{1}$-weighted sequences. Though DWI detected the additional number of metastatic lesions compared with the dynamic phase and hepatobiliary phase, imaging difference was not statistically significant. Results were thus discordant with the study of Löwenthal et $\mathrm{a}^{11}$ in which metastatic lesion detection rate was significantly higher for hepatobiliary phase compared with DWI and dynamic images. The discrepancy might be because of the limited number of the study population (22) in our prospective study, while a significantly large number of patients (73) were included in their retrospective study. Also, lesions evaluated in their study (332) were significantly more than that evaluated in our study (96).

The hepatobiliary phase was also found superior to $\mathrm{T}_{1}$-weighted and $\mathrm{T}_{2}$-weighted sequences in detecting liver metastases with a statistically significant difference between them. Our results were concordant with those of Caudana et $\mathrm{al}^{12}$ in which they reported that a significantly greater number of small metastases were detected with postcontrast gadolinium benzyloxypropionictetra-acetate images than unenhanced MRIs.

There were a few limitations to our study. DWI dataset included was only respiratory-triggered images that have superiority over breath-hold DWI for lesion detection, yet there were some limitations of the respiratory-triggered technique, like cardiac motion artifacts and noise contamination, which distorted ADC values to a certain degree. Adding the hepatobiliary phase of gadobenate (60-minute phase) to our study prolonged our examination study without adding significant advantage in detecting liver metastases compared with DWI.

\section{Conclusion}

The combination of DWI-MRI with gadobenate-enhanced $\mathrm{T}_{1}$-weighted MRI significantly improved the diagnostic accuracy of liver metastases detection and characterization compared with noncontrast MRI. The superiority of DWI in detecting small metastases ( $<10 \mathrm{~mm}$ in size) was also shown. Adding DWI to the routine MRI sequences is thus recommended in a patient of known primary malignancy to improve small metastases detection.

\section{Declaration of Patient Consent}

The authors certify that they have obtained all appropriate patient consent forms. In the form the patient (s) has/have given his/her/their consent for his/her/their images and other clinical information to be reported in the journal. The patients understand that their names and initials will not be published and due efforts will be made to conceal their identity, but anonymity cannot be guaranteed.

\section{Financial Support and Sponsorship}

Nil.

\section{Conflicts of Interest}

There are no conflicts of interest.

\section{References}

1 Ackerman NB, Lien WM, Kondi ES, Silverman NA. The blood supply of experimental liver metastases. I. The distribution of hepatic artery and portal vein blood to "small" and "large" tumors. Surgery 1969;66(6):1067-1072

2 Ward J. New MR techniques for the detection of liver metastases. Cancer Imaging 2006;6:33-42

3 Wu LM, Hu J, Gu HY, Hua J, Xu JR. Can diffusion-weighted magnetic resonance imaging (DW-MRI) alone be used as a reliable sequence for the preoperative detection and characterisation of hepatic metastases? A meta-analysis. Eur J Cancer 2013;49(3):572-584

4 Chen L, Zhang J, Zhang L, et al. Meta-analysis of gadoxetic acid disodium (Gd-EOB-DTPA)-enhanced magnetic resonance imaging for the detection of liver metastases. PLoS One 2012;7(11):e48681

$5 \mathrm{Kim} \mathrm{HJ}$, Lee SS, Byun JH, et al. Incremental value of liver MR imaging in patients with potentially curable colorectal hepatic metastasis detected at CT: a prospective comparison of diffusion-weighted imaging, gadoxetic acid-enhanced MR imaging, and a combination of both MR techniques. Radiology 2015;274(3):712-722

6 Muhi A, Ichikawa T, Motosugi U, et al. Diagnosis of colorectal hepatic metastases: comparison of contrast-enhanced CT, contrast-enhanced US, superparamagnetic iron oxide-enhanced MRI, and gadoxetic acid-enhanced MRI. J Magn Reson Imaging 2011;34(2):326-335

7 Vreugdenburg TD, Ma N, Duncan JK, Riitano D, Cameron AL, Maddern GJ. Comparative diagnostic accuracy of hepatocyte-specific gadoxetic acid (Gd-EOB-DTPA) enhanced MR imaging and contrast enhanced CT for the detection of liver metastases: a systematic review and meta-analysis. Int J Colorectal Dis 2016;31(11):1739-1749

8 Hagspiel KD, Neidl KF, Eichenberger AC, Weder W, Marincek B. Detection of liver metastases: comparison of superparamagnetic iron oxide-enhanced and unenhanced MR imaging at $1.5 \mathrm{~T}$ with dynamic CT, intraoperative US, and percutaneous US. Radiology 1995;196(2):471-478

9 Ichikawa T, Saito K, Yoshioka N, et al. Detection and characterization of focal liver lesions: a Japanese phase III, multicenter comparison between gadoxetic acid disodium-enhanced magnetic resonance imaging and contrast-enhanced computed tomography predominantly in patients with hepatocellular carcinoma and chronic liver disease. Invest Radiol 2010;45(3):133-141

10 Bruegel M, Gaa J, Waldt S, et al. Diagnosis of hepatic metastasis: Comparison of respiratory-triggered echo -planar MRI and five T2-weighted turbo spin-echo sequences. AJR Am J Roentgenol 2008;191:1421-1429

11 Löwenthal D, Zeile M, Lim WY, et al. Detection and characterisation of focal liver lesions in colorectal carcinoma patients: comparison of diffusion-weighted and Gd-EOB-DTPA enhanced MR imaging. Eur Radiol 2011;21(4):832-840

12 Caudana R, Morana G, Pirovano GP, et al. Focal malignant hepatic lesions: MR imaging enhanced with gadolinium benzyloxypropionictetra-acetate (BOPTA)-preliminary results of phase II clinical application. Radiology 1996;199(2):513-520 